\title{
MNGIE with lack of skeletal muscle involvement and a novel TP splice site mutation
}

\author{
K Szigeti, L-J C Wong, C-L Perng, G M Saifi, K Eldin, A M Adesina, D L Cass, M Hirano, J R Lupski, \\ F Scaglia
}

J Med Genet 2004;41:125-129. doi: 10.1136/jmg.2003.013789

\begin{abstract}
Mitochondrial neurogastrointestinal encephalomyopathy (MNGIE) is an autosomal recessive multisystem disorder caused by thymidine phosphorylase (TP) deficiency, resulting in severe gastrointestinal dysmotility and skeletal muscle abnormalities. A patient is reported with a classical MNGIE clinical presentation but without skeletal muscle involvement at morphological, enzymatic, or mitochondrial DNA level, though gastrointestinal myopathy was present. MNGIE was diagnosed by markedly raised plasma thymidine and reduced thymidine phosphorylase activity. Molecular genetic analysis showed a homozygous novel splice site mutation in TP. On immunohistochemical studies there was marked TP expression in the CNS, in contrast to what has been observed in rodents. It is important to examine the most significantly affected tissue and to measure TP activity and plasma thymidine in order to arrive at an accurate diagnosis in this condition.
\end{abstract}

M tochondrial neurogastrointestinal encephalomyopathy (MNGIE) is an autosomal recessive disorder caused by mutations in the thymidine phosphorylase gene (TP). ${ }^{1}$ It is a member of a growing number of autosomal recessive disorders classified as defects of intergenomic

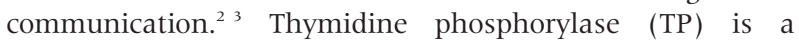
nuclear encoded enzyme catalysing phosphorolysis of thymidine to thymine and deoxyribose 1-phosphate. In normal cells thymidine is either degraded to thymine by $\mathrm{TP}$, or salvaged to deoxythymidine monophosphate by thymidine kinase. ${ }^{4}$ In MNGIE, lack of TP activity leads to accumulation of thymidine and deoxyuridine. Nucleoside and nucleotide pools are likely to be disturbed, resulting in mitochondrial DNA alterations, such as multiple deletions, depletion, and somatic point mutations. ${ }^{5}$ Mitochondria are more susceptible to the altered nucleoside pools because mtDNA constantly replicates, even in postmitotic cells.

Clinical diagnostic criteria have been established for MNGIE. ${ }^{6}$ The criteria include:

- severe gastrointestinal dysmotility presenting as early satiety, abdominal cramps, and diarrhoea, or in the more severe cases as repeated attacks of vomiting and pseudoobstruction which can lead to laparotomies and the need for parenteral nutrition;

- cachexia following the above;

- ptosis;

- external ophthalmoparesis;

- peripheral neuropathy;

- leucoencephalopathy.

Leucoencephalopathy on neuroimaging studies is predictive of the presence of TP mutations. ${ }^{4}$ Laboratory studies reveal raised thymidine and deoxyuridine levels (typically more than 100-fold above normal), decreased thymidine phosphorylase activity in leucocytes, and diminished thymidine clearance. ${ }^{78}$ Muscle biopsy has been abnormal in all reported cases. $^{6}$ Mitochondrial DNA mutation analysis showed multiple deletions or depletion in all patients with a confirmed mutation in the TP gene. ${ }^{6}$ Recently the presence of site specific somatic mtDNA point mutations in tissues and cultured cells from MNGIE patients has been demonstrated. ${ }^{5}$

Thus far, 25 distinct TP mutations have been reported, including 15 missense, six splice site, three microdeletions, and one single nucleotide insertion. ${ }^{69}$ Consistent with recessive inheritance, each patient was either homozygous or compound heterozygous for TP mutations, with demonstrated loss of function by measurement of TP activity in buffy coat samples.

\section{CASE REPORT}

A 17 year old white boy presented with acute abdominal pain. He had a six month history of progressive vomiting, inability to tolerate food, and $18 \mathrm{~kg}$ weight loss. Exploratory laparotomy did not identify an aetiology. He was subsequently maintained on parenteral nutrition. He developed a burning sensation in the feet, diplopia, and progressive weakness. The neurological examination was significant for external ophthalmoparesis, ptosis, diffuse weakness with a marked distal to proximal gradient, and absent reflexes in the legs. There was diminished sensation to all modes in a glove and stocking distribution, and allodynia.

Laboratory investigations showed a venous lactate concentration of $4.6 \mathrm{mmol} / \mathrm{l}$ (normal 0.2 to 2 ) with a pyruvate of $0.2 \mathrm{mg} / \mathrm{dl}$ (normal 0.3 to 0.9 ) and a lactate to pyruvate ratio of 23. Magnetic resonance imaging and magnetic resonance spectroscopy of the brain showed leucoencephalopathy and a cerebrospinal fluid lactate peak with abnormal small lactate peaks in the basal ganglia and temporal white matter. Electrophysiological testing revealed moderate to severe demyelinating and axonal sensorimotor neuropathy. A muscle biopsy was done as a diagnostic procedure. Mitochondrial DNA mutation analysis was undertaken on skeletal muscle and small intestine. Subsequently, serum thymidine concentration $(10.9 \mu \mathrm{M}$, normal $<0.05)$, deoxyuridine concentration $(15.6 \mu \mathrm{M}$, normal $<0.05)$, and TP activity (31 nmol/h/mg protein, normal $667 \pm 212$ ) were measured. Molecular genetic analysis of TP was done. The patient succumbed to pulmonary embolism and necropsy was carried out.

\section{MOLECULAR METHODS}

Blood samples were collected after informed consent. DNA was isolated from peripheral blood leucocytes by the salting out method. Mitochondrial DNA (mtDNA) point mutations, deletions, and depletion were analysed..$^{10}$ 
Cell lines were established by EBV transformation. RNA was isolated from the cell lines using the RNAeasy Mini Kit (Qiagen, Valencia, California, USA). Reverse transcription was accomplished using an oligo dT primer and superscript II (Invitrogen, Carlsbad, California, USA). PCR was carried out using the Ml3 tagged primers: MI3FGAAGGGAGCCAGGGACTTC and M13R-TCTGACCCACGAT ACAGCAG, in which MI3F and MI3R represent the universal DNA sequencing primers, TGTAAAACGACGGCCAGT and CAGGAAACAGCTATGACC, respectively. The reaction mix contained HotStarTaq (Qiagen) and was cycled 50 times at $60^{\circ} \mathrm{C}$ annealing. Sequencing was done using DyePrimer kit (ABI, Foster City, California, USA) on an ABI 377 sequencing machine. DNA sequence was analysed using Sequencher software (Genecodes, Ann Arbor, Michigan, USA) with splice sites examined by the neural network method (http:// www.fruitfly.org/seq.tools/splice.html).

Skeletal muscle biopsy and bowel resection and biopsy specimens were processed using standard clinical diagnostic protocols. Skeletal muscle mitochondrial enzyme activity was measured as a clinical diagnostic procedure using standard spectrophotometric analysis. Immunohistochemistry was done with anti-thymidine phosphorylase antibody (1:100) by the DAB detection method on the Ventana automated system.

\section{RESULTS AND DISCUSSION}

Sequencing of the coding regions and the adjacent splicing junctions showed the presence of a novel homozygous $\mathrm{G} \rightarrow \mathrm{C}$ transversion at the splice acceptor site of the second coding exon (designated IVSI-1G $>\mathrm{C}^{12}$; fig $\mathrm{lA}$ and $\mathrm{IB}$ ). Subsequently incest was disclosed. Splice site analysis predicts loss of the normal acceptor site, implicating skipping of this exon. RTPCR analysis followed by sequencing of the expected product did indeed show that the second coding exon was skipped (fig IC). However, it is not the normal splice donor site of the first coding exon that ligates to the splice acceptor site of the third coding exon. Instead, we find that an alternative splice donor site, 31 nucleotides upstream of the usual splice donor site, ligates to the splice acceptor site of the third coding exon. This suggests that the IVSI-lG $>C$ mutation either suppresses the normal splice donor site or unmasks an alternative cryptic splice donor site. Splice site prediction revealed a score of 0.09 (threshold 0.4) for the new splice site, indicating that unmasking may not be sufficient for the alternative splice donor site to activate. We hypothesise that the mutation most probably suppresses the normal splice donor site of the first coding exon.

The alternative splicing results in deletion of 234 nucleotides from the mRNA sequence which, by conceptual translation, leads to an in-frame deletion of 78 amino acids from the protein (fig 1D). The deleted amino acids span from residues 62 to 139 of the wild-type protein. TP enzyme crystallises as a dimer with identical subunits in Escherichia coli. Each subunit consists of two distinct domains: a small helical domain containing seven $\alpha$ helices and a larger $\alpha-\beta$ domain, and three regions connecting the two domains. A putative phosphate binding site is in the $\alpha-\beta$ domain, while the thymidine binding site is located in a cavity between the two domains. The substrate binding sites are near highly conserved regions ( $E$ coli residues 87-91, 110-135, and 163193). ${ }^{13}{ }^{14}$ Three highly conserved residues-His85, Arg171 and Lys 190-form the catalytic core. Site directed mutagenesis in these regions diminished TP activity. ${ }^{15} 16$ In the human, the corresponding residues of this catalytic triad are His116, Arg 202, and Lys221. In our patient, the deleted fragment contains the Hisll6 residue; thus its absence probably impairs function. The predicted loss of function was confirmed by the markedly reduced enzyme activity
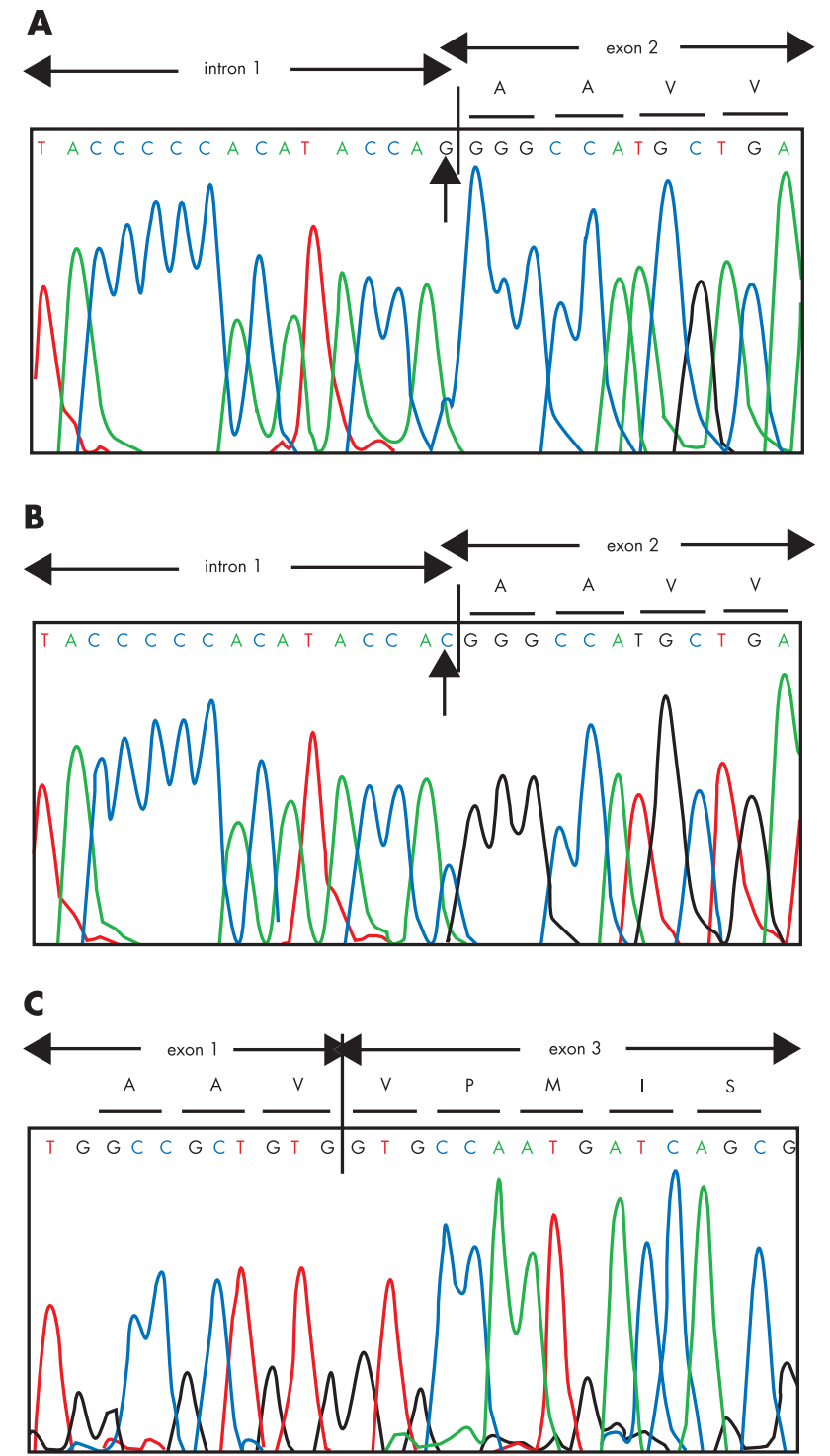

D

AAV VNGSAQGAQIGAMLMAIRLRGMDLEETSVLTQALAQSGQQ LEWPEAWRQQLVDKHSTGGVGDKVSLVLAPALAACGCKVPMIS

Figure 1 Thymidine phosphorylase (TP) mutation and its consequences on protein structure. (A) Normal DNA sequence. (B) The homozygous IVS1-1G >C mutation at the splice acceptor site of the second coding exon is indicated by a vertical arrow. (C) Reverse transcription polymerase chain reaction (PCR) followed by sequencing of the PCR product reveal that the $5^{\prime}$ part of the first coding exon ligates with exon 3. (D) Amino acid sequence of the deleted peptide (shown in italics) Sequences in bold are found in the mutant protein. For instance, the first three residues shown (AAV) are part of exon 1 (see (C)) and the last five residues (VPMIS) represent the beginning of exon 3 . The first 10 italicised residues (underlined) belong to coding exon 1; the remaining italicised residues (68) are from the skipped coding exon 2. His 116 (shown in red) is the a member of the catalytic triad.

measured in peripheral blood. Interestingly, as the deletion is in-frame, the protein preserved its antigenicity to the TP antibody, described below.

Mitochondrial DNA was analysed for deletions and depletion in muscle and for deletions in the small intestine, and none was detected. In the largest clinical study of MNGIE, involving 52 patients, partial depletion was found in 

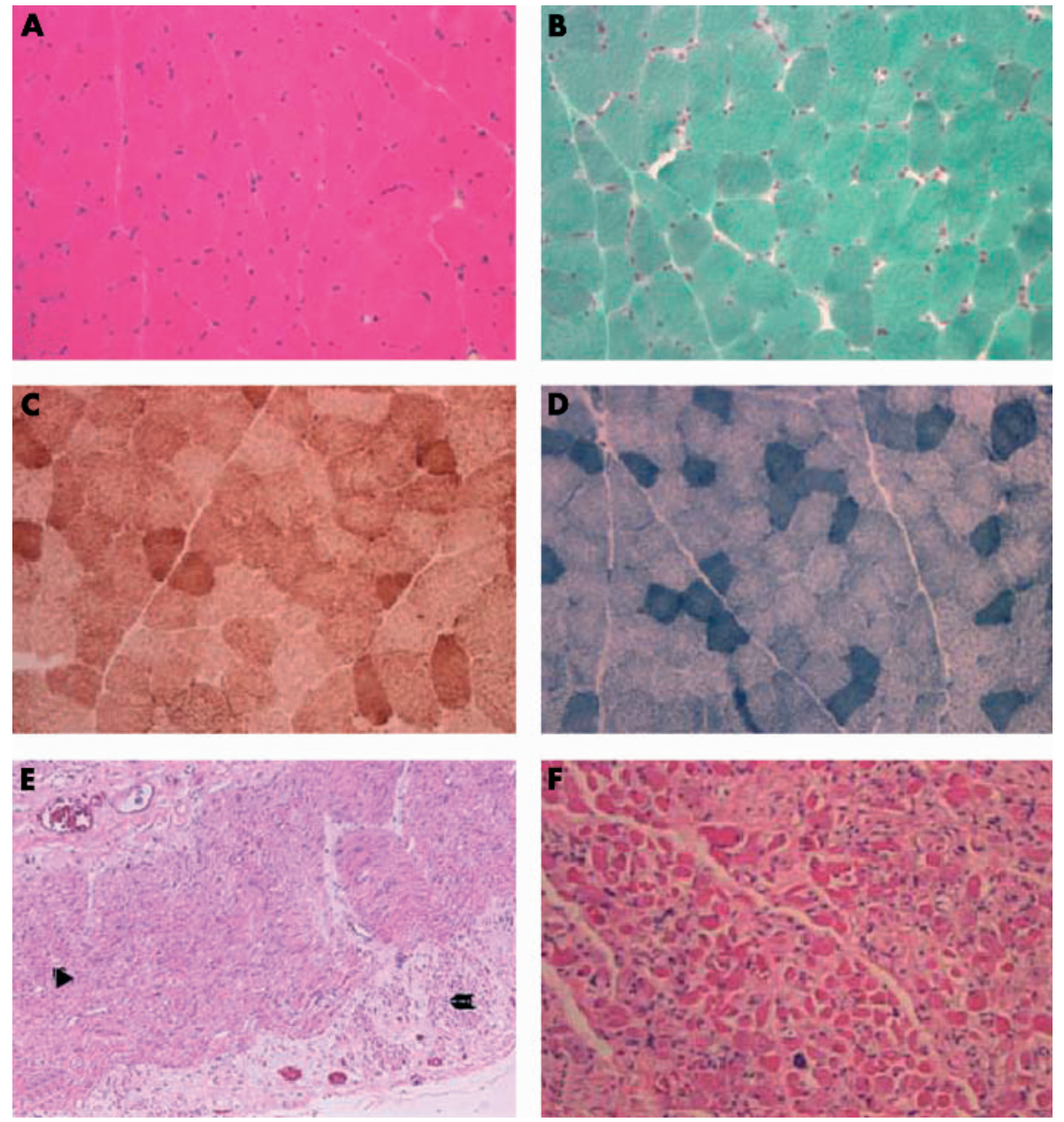

Figure 2 Muscle histopathology: pathological specimens showing normal skeletal muscle pathology on haematoxylin and eosin stain (A) and Gomori trichrome stain (B). There is normal COX (C) and NADPH activity (D) on muscle enzyme histochemistry. Gastrointestinal smooth muscle shows hypertrophied inner circular (arrowhead) and atrophied outer longitudinal layers (reverse arrowhead) (E). There is a severe myopathic pattern in the ocular muscle (F).

all the cases tested. ${ }^{6}$ In our case the most affected organ, the intestine, revealed smooth myopathy, but as there are no established standards for mtDNA content for the gut, depletion could not be assessed.

Muscle biopsy was obtained as a diagnostic study of choice to exclude or confirm mitochondrial disease. Muscle pathology was normal (fig 2A) except for glycogen accumulation, which was felt to be related to the chronic parenteral nutrition. Ragged red fibres were absent (fig 2B). Muscle enzyme histochemistry detected normal COX (fig 2C) and SDH activities and normal NADH (fig 2D). Electron microscopy showed unremarkable mitochondria. Previously, muscle biopsy was found to be abnormal in $100 \%$ of molecularly proven cases of MNGIE. All the cases had COX deficient fibres, while 63\% showed ragged red fibres or increased SDH staining. Muscle biochemical assay revealed normal activities of respiratory chain enzymes in our case, which can be consistent with MNGIE. ${ }^{6}$

As the patient underwent exploratory laparotomy we had the opportunity to evaluate the small bowel. Interestingly, the smooth muscle bundles of the muscularis propria, especially the inner circular layer, appeared to be thickened (fig 2E), and the smooth muscle was swollen, with pale cytoplasm. Some of the nerve fibres appeared enlarged and the number of ganglion cells was relatively sparse. The longitudinal layer of the muscularis propria was markedly atrophied.

At necropsy, there was a markedly myopathic pattern in the ocular muscles, with atrophied round fibres and fibrosis (fig 3F), providing evidence that myopathy rather than cranial neuropathy was responsible for the external ophthalmoplegia. The ocular muscles may be more prone to the effect of mitochondrial dysfunction as they have tonic activity, thus requiring a high energy supply. In this case, skeletal striated muscle, ocular tonic striated muscle, and smooth muscle in the gastrointestinal tract showed differential involvement at the microscopic level, correlating well with the clinical findings.

The in-frame deletion mutation preserved protein antigenicity and enabled us for the first time to assess TP regional expression patterns in the human central nervous system compared with that of a patient with MNGIE. Our observations allude to the pathomechanism of MNGIE associated CNS disease and may explain why the rodent model ${ }^{16}$ does not fully replicate the human disease. The cortex, basal ganglia, thalamus, midbrain, and cerebellum revealed a uniform cytoplasmic neuronal staining (fig 3C), similar to the normal control (fig 3A). The glial cells were negative for TP expression. Perivascular rod shaped cells, suggestive of microglia, were prominently reactive with the antibody. 

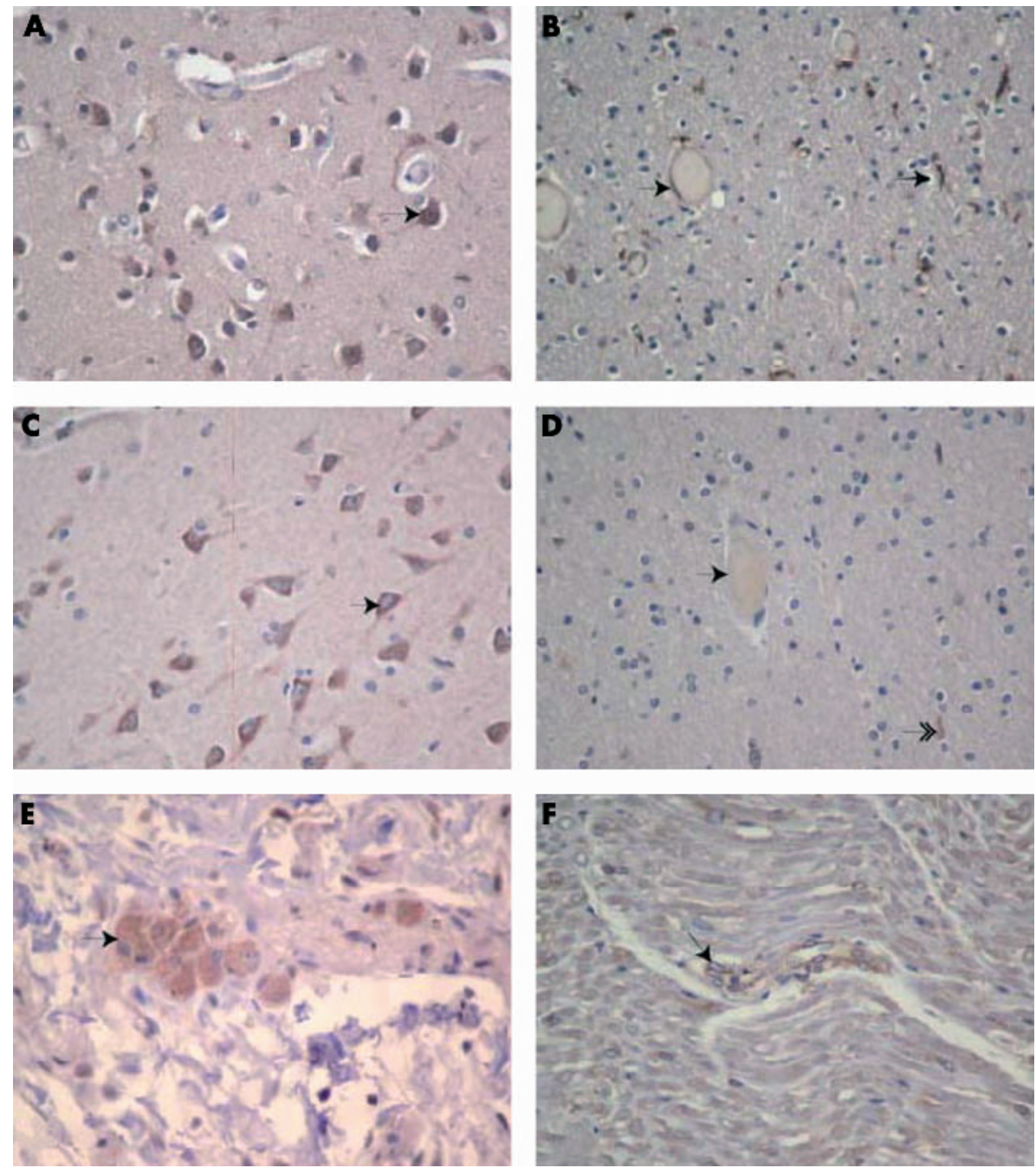

Figure 3 Thymidine phosphorylase (TP) expression by immunohistochemistry. Immunohistochemistry with anti-thymidine phosphorylase antibody reveals neuronal cytoplasmic staining (arrow) in the cortex of the patient (C) and normal control (A). The subcortical white matter of the control (B) shows endothelial and microglial expression (arrow), while the endothelial stain is markedly reduced in the patient, and microglial staining is diminished (D). Ectopic neurones (double arrow) serve as internal positive control in the patient's specimen. Jejunal section shows positive staining in the ganglion cells (E). Section of the cauda equina reveals endothelial and Schwann cell expression (F).

Interestingly, endothelial staining in the subcortical white matter showed decreased staining in the patient (fig 3D) compared with the normal control (fig 3B). This area of diminished TP endothelial staining corresponds to the white matter lesions observed on MRI antemortem. Pathological analysis did not show demyelination or gliosis in these areas; rather, the microscopic anatomy was preserved. We hypothesise that the abnormal signal on MRI indicates altered bloodbrain barrier permeability. The cauda equina showed TP expression in the Schwann cells and the endothelial cells. The GI tract revealed TP expression in the ganglionic cells and the endothelial cells, while TP was also present in the mucosa in a luminal to cryptic gradient in the small intestine and a reverse pattern in the stomach.

TP expression patterns have been studied previously in the rat. ${ }^{16}$ Rodent TP expression was present in all the neurones of the peripheral nervous system, but was lacking in the central nervous system and was very low in cultured Schwann cells. This marked difference in CNS TP expression between humans and rodents may explain in part the lack of CNS involvement in TP knockout mice. ${ }^{17}$

In our case the phenotype established the clinical diagnosis of MNGIE. The normal findings on muscle biopsy and lack of mitochondrial DNA depletion and multiple deletions confounded the picture, but the diagnosis was pursued because of the high index of suspicion. The MNGIE diagnosis was confirmed by demonstrating markedly decreased TP activity and raised serum concentrations of both thymidine and deoxyuridine. A novel TP splice acceptor site mutation was identified. If the clinical suspicion is high, lack of oxidative phosphorylation defects and normal histochemistry on skeletal muscle biopsy does not exclude the diagnosis of MNGIE.

\section{Authors' affiliations}

K Szigeti, G Mustafa Saifi, J R Lupski, F Scaglia, Department of Molecular and Human Genetics, Baylor College of Medicine and Texas Children's Hospital, Houston, Texas, USA

K Eldin, A M Adesina, Department of Pathology, Baylor College of Medicine and Texas Children's Hospital

D L Cass, Department of Surgery, Baylor College of Medicine and Texas Children's Hospital

L-J C Wong, C-L Perng, Institute for Molecular and Human Genetics, Georgetown University Medical Center, Washington DC, USA

M Hirano, Department of Neurology, Columbia University College of Physicians and Surgeons, New York, NY, USA 
Correspondence to: Dr James R Lupski, Baylor College of Medicine, One Baylor Plaza, Rm604B, Houston, Texas 77030, USA;

jlupski@bcm.tmc.edu

Received 26 August 2003

Accepted 19 September 2003

\section{REFERENCES}

1 Nishino I, Spinazzola A, Hirano M. Thymidine phosphorylase gene mutations in MNGIE, a human mitochondrial disorder. Science 1999;283:689-92.

2 Hirano M, Marti R, Ferreiro-Barros C, Vila MR, Tadesse S, Nishigaki Y, Nishino I, Vu TH. Defects of intergenomic communication: autosomal disorders that cause multiple deletions and depletion of mitochondrial DNA. Semin Cell Dev Biol 2001;12:417-27.

3 Zeviani M, Spinazzola A, Carelli V. Nuclear genes in mitochondrial disorders. Curr Opin Genet Dev 2003;13:262-70.

4 Nishino I, Spinazzola A, Hirano M. MNGIE: from nuclear DNA to mitochondrial DNA. Neuromuscul Disord 2001;11:7-10.

5 Nishigaki Y, Marti R, Copeland WC, Hirano M. Site-specific somatic MtDNA point mutations in patients with thymidine phosphorylase deficiency. J Clin Invest 2003;111:1913-21.

6 Hirano M, Nishigaki Y, Marti R. MNGIE: A disease of two genomes. Neurologist (in press)

7 Spinazzola A Marti R, Nishino I, Andreo AL, Naini A, Tadesse S, Pela I Zammarchi E, Donati MA, Oliver JA, Hirano M. Altered thymidine metabolism due to defects of thymidine phosphorylase. J Biol Chem 2002;277:4128-33.

8 Marti R, Nishigaki Y, Hirano M. Elevated plasma deoxyuridine in patients with thymidine phosphorylase deficiency. Biochem Biophys Res Commun 2003:303:14-18.
9 Kocaefe YC, Erdem S, Ozguc M, Tan E. Four novel thymidine phosphorylase gene mutations in mitochondrial neurogastrointestinal encephalomyopathy syndrome (MNGIE) patients. Eur J Hum Genet 2003;11:102-4

10 McComsey G, Tan DJ, Lederman M, Wilson E, Wong $\sqcup$. Analysis of the mitochondrial DNA genome in the peripheral blood leukocytes of HIV-infected patients with or without lipoatrophy. Aids 2002;16:513-18.

11 Liang MH, Wong $\amalg$. Yield of mtDNA mutation analysis in 2000 patients. Am J Med Genet 1998;77:395-400.

12 den Dunnen JT, Antonarakis SE. Mutation nomenclature extensions and suggestions to describe complex mutations: a discussion. Hum Mutat 2000;15:7-12

13 Rick SW, Abashkin YG, Hilderbrandt RL, Burt SK. Computational studies of the domain movement and the catalytic mechanism of thymidine phosphorylase. Proteins 1999;37:242-52.

14 Moghaddam A, Zhang HT, Fan TP, Hu DE, Lees VC, Turley H, Fox SB, Gatter KC, Harris AL, Bicknell R. Thymidine phosphorylase is angiogenic and promotes tumor growth. Proc Natl Acad Sci USA 1995;92:998-1002.

15 Miyadera K, Sumizawa T, Haraguchi M, Yoshida H, Konstanty W, Yamada Y, Akiyama S. Role of thymidine phosphorylase activity in the angiogenic effect of platelet derived endothelial cell growth factor/thymidine phosphorylase. Cancer Res 1995;55:1687-90.

16 Eccleston PA, Funa K, Heldin $\mathrm{CH}$. Neurons of the peripheral nervous system express thymidine phosphorylase. Neurosci Lett 1995;192:137-41.

17 Haraguchi M, Tsujimoto H, Fukushima M, Higuchi I, Kuribayashi $\mathrm{H}$, Utsumi $\mathrm{H}$, Nakayama A, Hashizume H, Hirato J, Yoshida H, Hara H, Hamano S, Kawaguchi H, Furukawa T, Miyazono K, Ishikawa F, Toyoshima H, Kaname T, Komatsu M, Chen ZS, Gotanda T, Tachiwada T, Sumizawa T, Miyadera K, Osame M, Yashida H, Noda T, Yamada Y, Akiyama S. Targeted deletion of both thymidine phosphorylase and uridine phosphorylase and consequent disorders in mice. Mol Cell Biol 2002;22:5212-21.

\section{BOOK REVIEW}

\section{Psychiatric genetics: methods and reviews}

Edited by M Leboyer and F Bellivier. Totowa, NJ: Humana Press, 2003, $£ 82.50$ (US\$99.50), pp 268. ISBN 1588290379

This book is timely. Psychiatric genetics, which is one of the most challenging areas of medical genetics, currently offers enormous opportunities to improve the understanding of mental illness and the practice of clinical psychiatry. Phenotypic issues are, of course, important in all aspects of complex genetics, and within psychiatric genetics issues of phenotype definition and measurement are crucial. Major molecular genetic investigations using both positional and candidate approaches have been ongoing for several of the major psychiatric disorders over the last 10 or 15 years. Early successes, of course, occurred for Alzheimer's disease and linkage regions are currently being followed up for schizophrenia, bipolar disorder, autism, and attention deficit hyperactivity disorder as well as several other common severe psychiatric disorders for which there is compelling evidence of the importance of genetic factors in contributing to pathogenesis. Robust findings are beginning to emerge and there is currently enormous interest from the pharmaceutical industry because the identification of susceptibility genes is likely to be a key stage in the development of novel targets and, subsequently, to lead on to the production of medications that are more effective and have fewer side effects than currently available treatments.

Psychiatric genetics: methods and reviews is an edited volume in the series on "Methods in molecular medicine" by Humana Press. It is a relatively compact volume that comprises 11 chapters with the main content divided into two sections: the first of these addresses methods currently used in psychiatric genetics and includes chapters on analytical methods, genetic markers, clinical assessment, and genetic association studies. The second section addresses new phenotypic approaches and includes chapters on alternative phenotypes, intermediate cognitive phenotypes associated with schizophrenia, biochemical endophenotypes in personality disorders, electrophysiological phenotypes, and imaging approaches to phenotype characterisation in genetic studies of schizophrenia. The chapter authors comprise an international line up of researchers most of whom are well known in the field of psychiatric genetics. Perhaps surprisingly, there is no coverage given to the current state of knowledge-either from classical genetic or molecular genetic studies-for any psychiatric or behavioural phenotypes.

The strength of this volume is that it provides a relatively succinct introduction to a range of issues relating to the field of psychiatric genetics. The section devoted to new phenotypic approaches is particularly useful in this regard. Undoubtedly this would be an excellent book for a young researcher about to enter the field of psychiatric genetics and would enable him or her to obtain a rapid overview of methodological issues. It will be of less interest to clinicians and researchers outside the field of psychiatric genetics. Such individuals would be likely to find it more useful either to read a book that addresses methodological issues more generally in complex genetics diseases-such as Analysis of multifactorial disease (Oxford: Bios Scientific Publishers Ltd, 2000), edited by Bishop and Sham, or alternatively to read a book which, in addition to dealing with basic methodological issues, also reviews the state of knowledge in a broad range of psychiatric disorders-such as Psychiatric genetics and genomics (Oxford: Oxford University Press, 2002) edited by McGuffin, Owen and Gottesman. 